\title{
Propuesta de las condiciones de las Aplicaciones móviles, para la construcción de un Entorno de Accesibilidad Personal para usuarios con discapacidad visual en las Smart Cities
}

\author{
Adrián López Delgado, Eva Olmedo, Pedro Tadeu y José Maria Fernández Batanero \\ ${ }^{*}$ ) Universidad de Granada - España, ${ }^{* *}$ Instituto Politécnico de Guarda - Portugal, ${ }^{(* *}$ ) Universidad de Sevilla - España
}

\begin{abstract}
RESUMEN
Los nuevos avances tecnológicos y el uso de nuevas aplicaciones móviles permiten acceder a múltiples posibilidades en las Smart Cities. Sin embargo, el uso de la tecnología no resuelve por sí misma problemas de inclusión de ciudadanos que, por sus condiciones sociales o personales, no pueden utilizarlas adecuadamente. También, estas personas quedan incapacitadas, aún más, para participar como ciudadanos activos en su ciudad. Creando nuevas barreras no arquitectónicas, sino tecnológicas, que son más difíciles de comprender. Este estudio emplea la observación directa de personas con baja visión o ceguera, para comprobar las dificultades y necesidades que presentan en el uso de distintas aplicaciones en los sistemas Android e iOS. Y como resultados se comprueba que todos ellos necesitan aplicaciones complementarias, que en muchos casos no son compatibles con las diseñadas para el resto de personas. Y que, por tanto, no pueden acceder a todos los recursos de la Smart City. Para solucionarlo se propone la creación de un Entorno de Accesibilidad Personal (PAE), a través del cual se le permite el acceso al conjunto de todos los contenidos necesarios para interactuar con el entorno en el que se encuentra.
\end{abstract}

Palabras Clave: Tiflotecnología, Smart City, metodología observacional, discapacidad visual, App

\section{Proposal of Mobile Applications conditions to develop a Personal Accessibility Environment for users with visual disabilities in Smart Cities}

\section{ABSTRACT}

New technological advances and use of new mobile applications facilitate a easy access to multiple possibilities in smart cities. However, the use of technology does not solve inclusion problems of citizens who, due to their social or personal conditions, cannot use them adequately. Also, these people are incapacitated, even more, to participate as active citizens in their city. Creating a new nonarchitectural barriers, but technological ones, which are more difficult to understand.

This study employs the direct observation of people with low vision or blindness, to check the difficulties and needs they present in the use of different applications from Android and iOS systems. And as results, it is verified that all of them need complementary applications, which in many cases are not compatible with those designed for other people. And therefore, they cannot access to all the resources in the smart city.

To solve it is proposed the creation of a Personal Accessibility Environment (PAE) to allow an access to a set of all necessary contents to interact with the environment in their locations.

Keywords: Tiflotechnology, Smart City, observational methodology, visual disability, App.

\section{Introducción}

Con la aparición de dispositivos y aplicaciones móviles y su influencia en la vida diaria de los ciudadanos, la manera de ver y entender lo que nos rodea ha cambiado. Cada usuario puede configurar su dispositivo móvil según sus necesidades; añadiendo nuevas aplicaciones, tanto de pago como gratuitas, ayudán- dole a sacar un mayor partido al dispositivo, y creando el propio Entorno Personal Móvil (PME, en adelante) del usuario.

Estas herramientas aportan beneficios importantes a nivel profesional, personal o social, convirtiéndose en un utensilio indispensable en el día a día de cualquier ciudadano. Sin embargo, cuando el acceso no es igualitario para todos los habitantes, surgen las barreras de acceso, ocasionado por la inaccesibilidad de estos productos. 
En este lado inadaptado de la ciudad se encuentran aquel colectivo que cuyas dificultades de origen sensorial, físicas, intelectuales o simplemente por dificultades económicas o de interacción. Estas dificultades que encuentran este colectivo no son inherentes a su propia persona, sino que dependen de diversas variables, entre ellas el entorno en el que se desarrolla.

La accesibilidad es un derecho que en estos últimos años se ha negado a gran parte de la ciudadanía, afectando sobre todo al colectivo con discapacidad, y en concreto a las minorías de la población citadas anteriormente (Olmedo-Moreno y López-Delgado, 2015).

El objetivo debería enfocarse en suprimir las barreras que pueden encontrarse, así como eliminar la brecha tecnológica que existe para los colectivos con algún tipo de diversidad funcional, focalizando la atención, por tanto, en la versatilidad que tienen las ciudades y las tecnologías en adaptarse a estos, la potencialidad de estas.

De la necesidad de desarrollar estrategias inclusivas para que todas las personas de un municipio se encuentren incluidos en el mismo, y evitar de esta manera diferencias entre personas, surge la idea de la Smart Human City_(Olmedo-Moreno y López-Delgado, 2015). Este es un proyecto de ciudad más humano, que la Smart City, ya que atiende al ejercicio de derechos, y como principal receptor a los individuos. Para lo que se requiere de la estrecha colaboración entre administración pública y empresas privadas a la hora de la creación de material accesible para el sector con discapacidad, en un trabajo conjunto que genere la creación y desarrollo de los servicios básicos con App accesibles, sin necesidad de buscar alternativas (Álvarez, 2015).

Se hace necesario, por tanto, pensar más en el habitante; en el ciudadano. Creando entornos accesibles para todos y rompiendo así con la brecha digital que germina en nuestras nuevas ciudades inteligentes. ¿Pero qué es exactamente una "App"? Bien, el término de "App" proviene de la palabra aplicación, definida por Gil y Rodríguez-Porrero (2013) como una aplicación informática incluida en dispositivos móviles. Estas aplicaciones han sido diseñadas especialmente para su articulación en móviles y tablets, cuyo acceso es a través de una pantalla táctil. Dichas aplicaciones se pueden descargar, de manera gratuita o de pago, de sitios online gestionados por la compañía del dispositivo, aunque las plataformas más comunes y utilizadas a nivel global es gestionada por la popular compañía Google, "Play Store" y Apple, "App Store".

Tenemos al alcance de nuestro Smartphone o Tablet un gran número de "Apps", sin embargo, estas aplicaciones que se enmarcan en el ámbito de las Smart Cities tienen que cubrir unas necesidades y características, necesitan ser accesibles para todos, teniendo como principal objetivo una inclusión tecnológica global, conseguir que las aplicaciones lleguen al mayor número de usuarios posible, sin dejar de lado a ningún colectivo de población.

Dentro del concepto de accesibilidad encontramos variantes que dependen del contexto al que refieren; accesibilidad web, de aplicaciones inteligentes..., entre otras. En este caso, como estamos hablando de ciudades inteligentes y en ellas vemos implicadas las tecnologías de la información y comunicación, es interesante definir el concepto de accesibilidad web que está estrechamente ligado al de aplicación inteligente. Para ello, Hassan y Martín (2003) define la accesibilidad web como la "posibilidad de que un producto o servicio web pueda ser accedido y usado por el mayor número posible de personas, indiferentemente de las limitaciones propias del individuo o de las derivadas del contexto de uso "(p. 1).

Más centrado en el estudio planteado, entendemos por App accesible aquella en la que el usuario puede interactuar con los elementos de la aplicación independientemente de que éste sufra una discapacidad física, psíquica, sensorial o alguna otra carencia.

Al hablar de "elementos de la aplicación", nos referimos a los elementos de la interfaz de la aplicación, que engloba la vista genérica al iniciar la aplicación, los controles para acceder a ella (Gil y Rodríguez-Porrero, 2013).

A la hora de hablar de accesibilidad en una App, no sólo podemos referirnos a que el usuario pueda abrir o no la aplicación o pueda desenvolverse por el menú principal, sino a otros aspectos de la interfaz que pueden presentar barreras para algunos usuarios. Gil y Rodríguez-Porrero (2013) exponen otros aspectos relacionados con el diseño de la interfaz, como son la redacción de los mensajes, la organización en sí de la aplicación o los aspectos visuales como son el color, el contraste, entre otros. Todos estos aspectos deben cuidarse por parte del diseñador, ya que son aspectos básicos que dificultan mucho la accesibilidad y usabilidad de la aplicación.

Llegados a este punto nos podemos preguntar cuáles son los beneficios que las personas con dificultades de accesos pueden obtener de los productos tecnológicos. Pues bien, estos son inmensurables, y además muy fácil de conseguir, ya que con el simple hecho de que desarrolladores, previamente, hagan compatibles las herramientas de accesibilidad que incorporan los dispositivos móviles con la interfaz del producto en cuestión, conseguiríamos que la totalidad de usuarios que pueden interaccionar con el móvil pudieran hacerlo con las App.

La mayoría de la población encuentra en los Smartphone un medio de desarrollo de la autonomía del usuario, de sus capacidades y aptitudes, aumento de su integración social, su participación social como habitante en la ciudad, aumento de la autoestima y autoconcepto del mismo, en general, alcanzar a sentirse ciudadanos, sin tener obstáculos que lo impidan. Concretamente para aquellos usuarios con diversidad funcional, las tecnologías les abre un nuevo mundo de posibilidades:

- Permiten a sus usuarios estar comunicados en todo momento, estén donde estén. El disponer de un dispositivo móvil consigo mismo, permite a estos usuarios a sentirse acompañados y seguros, pudiendo ser atendidos en hechos puntuales o si acontece algún contratiempo, sintiéndose dependientes. Además, posibilita la creación de redes de contacto con otros usuarios.

- Integración, desarrollo y autonomía en el entorno. El desarrollo de sentimientos de competencia en un contexto tecnificado provocará en el usuario a sentirse parte de él, a ser capaz de interaccionar con el medio, y por lo tanto a que aumente su autoestima y autoconcepto al sentirse incluido.

- Aportan un entorno de ocio. Hoy día en los tiempos en los que corre, una de las ofertas de ocio más extendidas son las redes sociales y la navegación web para búsqueda de recursos audiovisuales a gusto del usuario, etc. Para el colectivo con algún tipo de dificultad en estos medios, es fundamental el paliar estos obstáculos para poder acceder a los contenidos que las TIC proporcionan.

- Medio informativo eficaz, personal y real. Una gran parte del sector de población afectado con cualquier tipo de discapacidad, acostumbra a recibir la información que a él le llega de forma física, o a consumir contenido que él recibe de forma involuntaria a través de los medios audiovisuales. Por ello, es fundamental que sean capaces de acceder a la información en red, pudiendo discriminar antes de consumir el contenido que se expone. 
- Entorno laboral. Hoy día, la mayoría de ofertas laborales se ofrecen de forma virtual, siendo necesario de disponer de un currículum vitae electrónico y acceso a la rede para poder cumplimentar cualquier oferta, y sentirte competente en este ámbito.

Como podemos ver en los anteriores motivos, el crear tecnologías accesibles debería ser una obligación, privando lo menos posible al colectivo al que referimos. Ello implica colaboración directa entre desarrolladores, fijar como rutina el añadir las directrices necesarias de accesibilidad ante la creación de un nuevo producto, aumentando la calidad de los servicios y la satisfacción de sus usuarios.

\section{La accesibilidad y la deficiencia visual}

La Organización Mundial de la Salud (2014) en su nota descriptiva número 282 divide la capacidad de visión en 4 niveles: La visión normal, la discapacidad visual moderada, la discapacidad visual grave y la ceguera. Agrupando la antepenúltima y la penúltima en el descriptivo de "baja visión", que junto al término de ceguera representan los casos de discapacidad visual.

Por lo tanto, podemos considerar la discapacidad visual como aquella alteración del sentido de la vista que dificulta la forma en la que el individuo percibe el mundo que le rodea. Cuando la pérdida de visión es total, hablaremos de ceguera, sin embargo, cuando la pérdida sea parcial, nos referiremos entonces a baja visión.

El órgano de la vista es el sentido que más información nos aporta; a través de la visión percibimos la realidad tal y como se muestra, dándonos la posibilidad de interactuar con el mundo que nos rodea. Desde la escuela se trata estas desventajas con equidad, tratando de compensar las desigualdades que se dan a nivel social y personal, entre otras. Es toda una lucha que implica un trabajo continuo por parte de los afectados como por parte del colectivo de profesionales que atiende a las desigualdades.

El 26 de octubre de 2016 se publicó una directiva de origen europeo sobre la accesibilidad de los sitios web y las aplicaciones móviles de organismos del sector público, esta entró en vigor en diciembre del 2016. Su objetivo principal es garantizar que aquellos usuarios con algún tipo de discapacidad o impedimentos de cualquier índole puedan acceder de manera productiva y en igualdad de condiciones a las TIC. Dir. 2016/2102 del Consejo, de 26 de octubre de 2018 expone lo siguiente:

La presente Directiva establece las normas por las que se exige a los Estados miembros que garanticen que los sitios web, independientemente del dispositivo empleado para acceder a ellos, y las aplicaciones para dispositivos móviles de los organismos del sector público cumplan los requisitos de accesibilidad.

Los estados miembros disponen hasta el 23 de septiembre de 2018 para traspasar esta directiva europea a su legislación sino es así, se puede incoar una infracción contra el país. Tras la aprobación de la ley se procederá a alcanzar los objetivos que la directiva detalla, previendo que los sitios web nuevos, desde que salga la ley en España, tengan un año de margen para que sean adaptados, y que el resto de sitios web tengan dos años de margen para que sean adaptados en función de las directrices que se detallen. Por otro lado, las aplicaciones para dispositivos móviles tendrán 3 años para hacerlas accesibles a toda la población.

Por lo tanto, según las instrucciones que detalla la directiva, para mediados del año 2021, las aplicaciones móviles de organismos del sector público serán accesibles.

A pesar de que la Dir. 2016/2102 del Consejo, de 26 de octubre de 2018 únicamente refiere a aquellas plataformas que han sido desarrolladas por organismos públicos, esta anima a los estados miembros en su artículo 34 y 35 a que amplíen el grado de aplicación a las entidades privadas.

\section{Tiflotecnología Móvil. Apps accesibles para el usuario}

La tiflotecnología se viene utilizando años atrás, para referir a aquellos sistemas portátiles de almacenamiento y procesamiento de la información, dispositivos de reproducción y grabación, impresoras braille, calculadoras con voz incorporada, sistemas de reconocimiento óptico (OCR), diccionarios y traductores con voz, entre otros, un conjunto de herramientas que permiten la adaptación para usuarios con deficiencias visuales.

En este escenario surgen distintas aplicaciones informáticas, para que los usuarios invidentes puedan operar con esta tecnología creada, implicando una formación desde edades tempranas para el entrenamiento y control eficaz de estos medios.

Según Cebrián de Miguel (2005), en su glosario de Tecnologías de la Información y la Comunicación, define la Tiflotecnología como:

Conjunto de técnicas, conocimientos y recursos que facilitan o proporcionan los medios oportunos, instrumentos auxiliares, ayudas o adaptaciones tecnológicas, creadas o adaptadas específicamente para posibilitar a las personas discapacitadas visuales y sordo-ciegas, la correcta utilización de la tecnología que contribuye a su autonomía personal y plena integración social, laboral y educativa.

En pleno año 2018, donde los dispositivos móviles cubren casi todas las necesidades que anteriormente los ordenadores de sobremesa o portátiles cubrían, la Tiflotecnología engloba un área más, incorporando los Smartphone y Tablet como objetivo a alcanzar, por tanto, podríamos denominar esta nueva área Tiflotecnología Móvil, en la que se incorpora el conjunto de herramientas y aplicaciones que facilitan el acceso e interacción entre usuario y dispositivo móvil, específicamente para posibilitar que aquellos usuarios con deficiencias visuales, de cualquier tipo, accedan a la tecnología de manera satisfactoria.

Estas herramientas y aplicaciones forman el Entorno Personal de Accesibilidad de cada usuario en particular, puesto que, según sus características o condiciones personales de cada usuario, las necesidades serán distintas unas de otras.

Para considerar una aplicación móvil accesible, los elementos de su interfaz deben ser accesibles para cualquier usuario que intente interaccionar con la App, pudiendo extraer de ella la información que anda buscando. En este proceso, el intercambio no es únicamente entre la interfaz de la aplicación y el usuario, sino que en el momento que haya necesidades específicas de accesibilidad, un tercer agente entra en juego: el Entorno Personal de Accesibilidad.

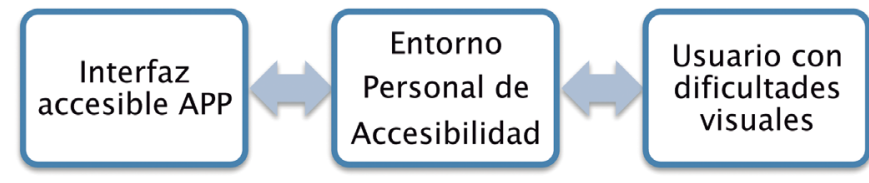

Figura 1. Proceso de una App accesible

En la figura 1, podemos observar el proceso que realiza un usuario con dificultades de visión a la hora de acceder a una aplicación móvil, la cual dispone de elementos accesibles en ella. Hablamos de un proceso interactivo en tanto en cuanto el usuario accede a su Entorno Personal de Accesibilidad para que el conjunto de herramientas o ayudas técnicas que lo componen, 
obtengan la información que alberga la App y que si no fuera de esta forma, por las dificultades tan específicas del usuario, no pudiese acceder al contenido.

Entendemos ayuda técnica como los productos, instrumentos, equipos o sistemas adaptados que usuarios con cualquier tipo de discapacidad necesita para equiparar o compensar sus capacidades personales, siendo creadas con intención específica para el colectivo (Cebrián de Miguel, 2005).

Del mismo modo, al igual que es un proceso interactivo, también es un proceso lineal, de forma que, para recibir el contenido expuesto, el usuario tiene que interaccionar en primer lugar con su Entorno Personal de Accesibilidad y éste con la información de la App, haciendo todo el proceso de forma contraria para que finalmente el usuario reciba el feedback de la App.

Hablamos de Entornos Personales de Accesibilidad (PAE) como aquel conjunto de herramientas o software que un usuario de dispositivos móviles o sitios web tiene que utilizar complementariamente $\mathrm{o}$, en ocasiones, exclusivamente, para poder acceder al contenido que en este se expone.

Actualmente, la gran revolución tecnológica ha hecho más dependiente a la población de dispositivos móviles para poder controlar o desenvolverse de manera óptima, ya no sólo para moverse por la ciudad, sino también para viajar, para navegar por internet, para organizar el tiempo de ocio, etc. Todo ello ha provocado la aparición de aplicaciones móviles que sacien la necesidad de información, así como la invención de dispositivos cada vez más capaces.

Un usuario sin dificultades para el manejo de esta tecnología simplemente tiene que acceder a esas plataformas y recibir la información que busca a través de estas. Sin embargo, aquellos usuarios que sus características personales no le permiten interaccionar con estos sistemas de la misma forma que un usuario sin dificultades lo hace, es fundamental que además de tener su entorno personal móvil, compuesto por un sinfín de aplicaciones y plataformas donde el usuario extrae información del entorno en el que se desarrolla, necesite su propio Entorno Personal de Accesibilidad (PAE), mediante el cual, con una combinación personal de herramientas, pueda acceder por mediación con su propio Entorno Personal Móvil (PME). Posibilitando un acceso lo más igualitario posible a la información.

Este conjunto de herramientas de las que se habla, engloba tanto las herramientas o ayudas técnicas que los dispositivos móviles incorporan con su sistema operativo para que aquellos usuarios con dificultades en visión, audición, en habilidades físicas y motoras, o con problemas en cuanto a lectoescritura y aprendizaje, entre otras, puedan interactuar de forma bidireccional con el dispositivo en cuestión; también, se refiere a aquellos programas desarrollados para que complementen al programa principal en sitios web o en ordenadores: magnificadores y lectores de pantalla, como por ejemplo JAWS, Orca, VoiceOver, Zoomtext, etc.

Además de todas estas herramientas, en ocasiones, la falta de accesibilidad de las aplicaciones o plataformas hace necesario el tener que descargar o instalar una versión accesible de la misma, siendo insuficientes aquellas herramientas complementarias, y teniendo que buscar alternativas para acceder a la información.

Dentro de los entornos personales de accesibilidad del sector de población con dificultades en manejo, comprensión e interacción de esta tecnología, encontramos 4 tipos de alternativas ante estas situaciones:

- Ayudas técnicas del Sistema Operativo con intención de transmitir el contenido que se presenta por un medio o a través de un mensaje que sea comprensible para el usua-

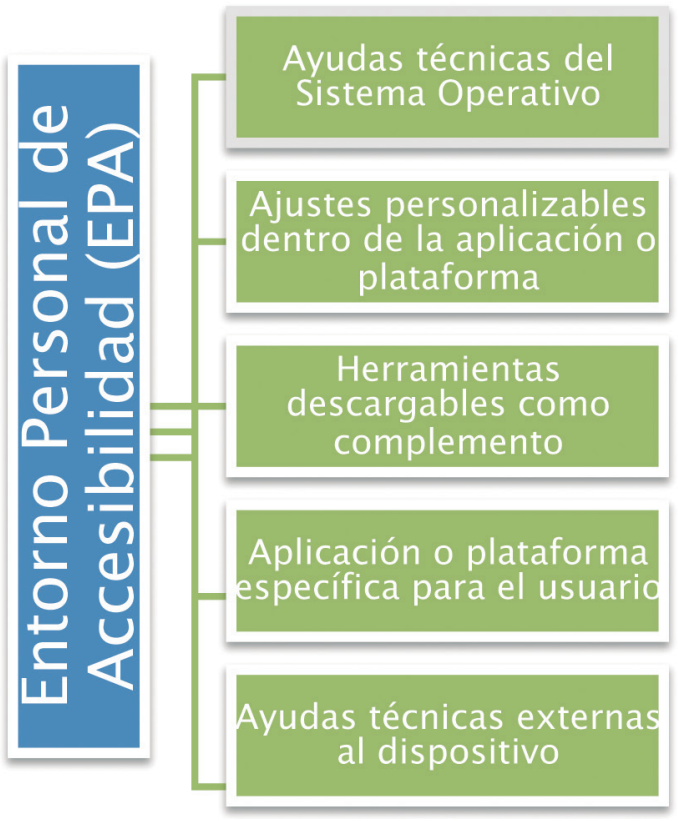

Figura 2. Posibilidades del Entorno Personal de Accesibilidad

rio en cuestión. Estas herramientas recogen todos aquellos ajustes que los dispositivos móviles incorporan en los dispositivos móviles.

- Ajustes personalizables dentro de la aplicación o plataforma. Incluye todas las modificaciones que los sitios web o aplicaciones móviles permiten realizar dentro, ayudando a que la experiencia del usuario sea algo más ajustada a las necesidades específicas que se demandan.

- Herramientas descargables como complemento. Recogen todos aquellos programas o aplicaciones que no vienen incorporadas en la tecnología y que se hace necesario para que pueda haber interacción entre aparato y usuario. Enfocados a dispositivos de mesa encontramos magnificadores y lectores de pantalla, como por ejemplo JAWS, Orca, Zoomtext, etc. En relación con los dispositivos móviles encontramos lectores de pantalla, conversores de pantalla, escritura por voz..., un gran abanico de aplicaciones para las necesidades puntuales de la población.

- Aplicación o plataforma específica para el usuario. Con intención de dar respuesta a las necesidades de aquellos usuarios con dificultades en visión, audición, en habilidades físicas y motoras, o con problemas en cuanto a lectoescritura y aprendizaje, entre otras, y debido a la carencia en cuanto a accesibilidad y usabilidad de algunas aplicaciones móviles, se crean aplicaciones que satisfagan esas necesidades, a través de aplicaciones alternativas a la de origen, como por ejemplo la aplicación "Metrociego" la cual es una alternativa a la aplicación de metro de la ciudad de Madrid.

- Ayudas técnicas externas al dispositivo. Para aquellos usuarios que necesiten o prefieran en ocasiones puntuales el uso de medios accesibles para recibir o introducir información de la aplicación, como por ejemplo líneas brailles (dependen de los revisores de pantalla), auriculares adaptados, micrófonos, etc.

Como hemos visto anteriormente, encontramos 5 formas distintas de acceder a todo el conjunto de datos que expone 
cualquier aplicación. Sin embargo, para poder considerar a una aplicación accesible como tal, debe permitir que las ayudas técnicas del Sistema Operativo recojan la información necesaria de la App para gestionar así las funciones que demanda el usuario.

No hablamos de crear aplicaciones con cientos de herramientas que la hagan accesibles, únicamente se busca la compatibilidad de la que hablamos, por ejemplo, que la ayuda técnica sea capaz de leer las funciones de los controles, y para ello tiene que haber un etiquetado correcto. Por lo tanto, para considerar una aplicación móvil accesible, únicamente, los desarrolladores deben ser capaces de incorporar en sus productos las directrices pertinentes que hagan compatible la interacción entre ayuda técnica y aplicación móvil.

\section{Finalidad y problema de investigación}

Este estudio se quiere realizar una selección de aplicaciones inteligentes enmarcadas en el contexto de las Smart Cities, analizando si estas son accesibles y usables para el usuario con discapacidad visual. A fin de conocer si existe inclusión digital para este colectivo al que referimos. Con lo que nos preguntamos si ¿Las aplicaciones inteligentes integran elementos accesibles y usables en su interfaz para ser consideras como tal para el usuario con discapacidad visual en el contexto de una ciudad inteligente?

Con objeto de dar respuesta a la cuestión planteada nos proponemos los siguientes objetivos:

1. Analizar el grado de accesibilidad y usabilidad, por los usuarios, del conjunto de aplicaciones seleccionadas como potenciales para la Smart City en los sistemas operativos iOS y Android.

2. Describir el Entorno Personal de Accesibilidad (Personal Accesibility Environment) en aquellos usuarios con deficiencias visuales.

3. Diseñar un protocolo de recomendaciones con principios y directrices para que las Apps sean accesibles a usuarios con deficiencias visuales.

\section{Metodología}

El estudio se realiza desde una metodología observacional directa participante, facilitado la interpretación y comprensión de las situaciones analizadas. La observación se ha registrado a través de la escala de estimación descriptiva construida ad hoc, sistema categoriales emergentes, registro anecdótico y notas de campo.

\subsection{Muestra}

El proceso de muestro ha sido polietápico dado que se ha trabajado con dos muestras diferenciadas por dos etapas de muestreo:

\section{Etapa 1}

Selección intencional de las aplicaciones inteligentes para dispositivos móviles que se encuentran en las plataformas de descargas más populares, en este caso, "App Store" y "Play Store". La muestra es el conjunto de aplicaciones seleccionadas como potenciales para el desarrollo de una Smart City, además de ser estas las que se sitúan en los primeros puestos de los rankings de Google Play y App Store en cuanto a las más descargadas a nivel mundial. Además se han tenido en consideración los criterios de:

- Funcionalidad de uso en la ciudad

- Proyección en el futuro

- Atención a Necesidades Específicas

- Compatibilidad

Como observamos en la tabla 1, la muestra seleccionada para el estudio es representada por un total de 16 aplicaciones, que son enmarcadas en 5 ámbitos, considerados elementales para su clasificación.

Tabla 1.

Muestra de las Apps seleccionadas en el estudio, agrupadas por entornos

\begin{tabular}{|c|c|c|c|c|}
\hline EDUCACIÓN & SOCIAL & SALUD Y DEPORTE & VIAJES Y TRANSPORTE & CONSUMO \\
\hline Dropbox & Facebook & Runtastic & Blablacar & JustEat \\
\hline Skype & InfoJobs & SMSCitaPrevia & Google Maps & Amazon \\
\hline \multirow[t]{3}{*}{ Duolingo } & WhatsApp & & MyTaxi & Wallapop \\
\hline & & & Uber & \\
\hline & & & Airbnb & \\
\hline
\end{tabular}

\section{Etapa 2}

Con un muestro por voluntarios, se han seleccionado a ciudadanos con deficiencias visuales, tanto ceguera como baja visión. Estos usuarios han sido seleccionados mediante entidades especializadas en deficiencias visuales, así como jornadas específicas del ámbito en cuestión. Estas son:

- ONCE (Organización nacional de ciegos españoles) de la ciudad de Cartagena

- ONCE de la ciudad de Murcia

- ADISVARM (Asociación de discapacitados visuales por la accesibilidad de la Región de Murcia)
- "X Jornadas de ASPREH", "Diversidad en las Capacidades Visuales"

Finalmente, la muestra seleccionada que podemos ver en la tabla 2, asciende a un total de 22 usuarios con deficiencias visuales variadas. Estos usuarios son los encargados de testear la muestra de las aplicaciones detalladas anteriormente.

Tabla 2.

Muestra de los usuarios seleccionados para el estudio

\begin{tabular}{lccc} 
MUESTRA & CEGUERA & 12 \\
$\begin{array}{c}\text { TOTAL PARA } \\
\text { TESTEO }\end{array}$ & 22 & BAJA VISIÓN & 11 \\
\hline
\end{tabular}




\subsection{Instrumentos}

En cuanto a los instrumentos de corte cuantitativo que se han utilizado, contamos con los siguientes:

- Se ha construido una Escala de Estimación Descriptiva, a modo de tabla de doble entrada donde las columnas se corresponden con los indicadores de accesibilidad a evaluar y, las filas con los propios niveles de accesibilidad que puede presentar. Esta ha sido validada mediante juicio de expertos.

- Se ha diseñado también un sistema de descriptores o de indicadores, compuesto por un total de 19 elementos, cuyo fin no es otro que detallar cuáles son los puntos a analizar en cada una de las aplicaciones de la muestra, obteniendo finalmente una valoración numérica del comportamiento de estos indicadores en la App en cuestión.

- Las notas de campo, cuya finalidad es recabar información relativa a las entrevistas realizadas con los profesionales elegidos para la validación mediante juicio de expertos. Estas han sido anotadas en el momento de la actuación y redactadas más extensamente tras la finalización de la entrevista.

- El registro anecdótico en la observación directa para registrar aquellas conductas o causas que consideramos re- levantes durante la realización del testeo o entrevista con los usuarios.

\section{Resultados}

Para el análisis de los datos recogidos se han interrelacionado, mediante el programa estadístico SPSS versión 25, las 16 variables de los indicadores de accesibilidad, a su vez, con las 18 variables de las Apps seleccionadas. Del mismo modo, las dos variables de los sistemas operativos ha sido necesario introducirlas, para conocer, de forma minuciosa y detallada, cómo se comportan cada uno de los indicadores, en base a las App y, en cada uno de los sistemas operativos predominantes en la sociedad actual.

En la siguiente tabla, podemos ver las variables detalladas anteriormente que, para poder valorar cada indicador, según su comportamiento en cada una de las apps, se ha pasado las respuestas a una escala 1 sobre 10, otorgando un valor a cada uno de los criterios de los ítems (0-5-10), siendo posible realizar una media aritmética $y$, un mayor tratamiento final de los resultados.

Como investigadores, nos interesa poder valorar cuantitativamente cada una de las Apps seleccionadas en la muestra en relación a la accesibilidad que presentan para aquellos usuarios con deficiencia visual.

Tabla 3.

Resultados obtenidos mediante el análisis experiencial de la muestra de población afectada por deficiencia visual seleccionada.

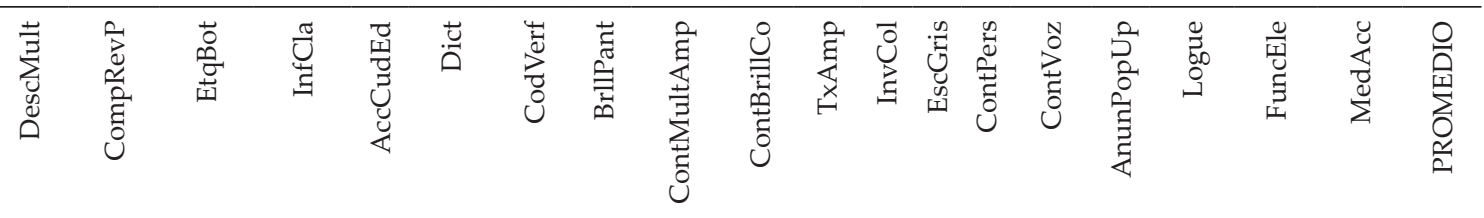

\begin{tabular}{|c|c|c|c|c|c|c|c|c|c|c|c|c|c|c|c|c|c|c|c|c|c|}
\hline \multirow{2}{*}{ Dropbox } & Android & 0 & 10 & 5 & 10 & 10 & 10 & - & * & 10 & * & 10 & * & * & * & 5 & - & 10 & 10 & 0 & 7,5 \\
\hline & iOS & 0 & 8,75 & 7,5 & 7,5 & 10 & 10 & - & * & 10 & * & 10 & * & * & * & 5 & - & 6,25 & 8,75 & 0 & 6,98 \\
\hline Skype & Android & 0 & 10 & 10 & 10 & 10 & 10 & - & * & 10 & * & 10 & * & * & * & * & - & 10 & 10 & 0 & 8,18 \\
\hline \multirow{2}{*}{ Duolingo } & Android & 0 & 10 & 5 & 10 & 10 & 10 & - & * & 10 & * & 10 & * & * & * & * & 10 & 10 & 10 & 2,5 & 8,13 \\
\hline & iOS & 0 & 10 & 10 & 10 & 10 & 10 & - & 10 & 10 & * & 10 & * & * & $*$ & 5 & - & 10 & 10 & 0 & 8,08 \\
\hline \multirow{2}{*}{ InfoJobs } & Android & 0 & 10 & 10 & 10 & 10 & 10 & - & * & 10 & 10 & 10 & * & * & * & * & 10 & 10 & 10 & 0 & 8,46 \\
\hline & iOS & 0 & 10 & 10 & 10 & 10 & 10 & - & 10 & 10 & * & 10 & * & * & * & 5 & - & 10 & 10 & 0 & 8,08 \\
\hline \multirow{2}{*}{$\begin{array}{r}\text { WhatsA- } \\
\text { pp }\end{array}$} & Android & * & 10 & 10 & 10 & 10 & 10 & 10 & * & 10 & * & 10 & * & * & * & 10 & - & 10 & 10 & 5 & 9,58 \\
\hline & iOS & 2,5 & 10 & 10 & 10 & 10 & 10 & 10 & 10 & 10 & 10 & 10 & * & * & * & 10 & - & 9 & 10 & 0 & 8,77 \\
\hline $\begin{array}{c}\text { CitaPre- } \\
\text { viaSMS }\end{array}$ & iOS & - & 10 & 10 & 10 & 10 & 10 & - & * & 10 & * & 10 & $*$ & * & $*$ & 5 & - & 10 & 10 & 0 & 8,64 \\
\hline \multirow{2}{*}{ Blablacar } & Android & - & 10 & 5 & 10 & 10 & 10 & - & * & - & * & 10 & * & * & * & 5 & - & 7,5 & 5 & 0 & 7,25 \\
\hline & iOS & - & 8,75 & 8,75 & 8,75 & 10 & 10 & - & 10 & - & * & 10 & $*$ & * & * & 5 & - & 8,75 & 8,75 & 0 & 8,07 \\
\hline \multirow{2}{*}{$\begin{array}{r}\text { Google } \\
\text { Maps }\end{array}$} & Android & 0 & 10 & 10 & 10 & 10 & 10 & - & * & 10 & * & 10 & * & $*$ & * & 5 & - & 10 & 10 & 2,5 & 8,13 \\
\hline & $\mathrm{iOS}$ & 0 & 10 & 10 & 10 & 10 & 10 & - & $*$ & * & * & 10 & $*$ & * & * & 5 & - & 10 & 10 & 0 & 7,73 \\
\hline \multirow{2}{*}{ MyTaxi } & Android & - & 10 & 5 & 10 & 10 & 10 & 0 & $*$ & 10 & * & 10 & * & * & * & 5 & - & 10 & 10 & 0 & 7,5 \\
\hline & iOS & - & 10 & 5 & 10 & 10 & 10 & 5 & * & 10 & * & 10 & * & * & * & 10 & - & 10 & 10 & 0 & 8,33 \\
\hline
\end{tabular}




\begin{tabular}{|c|c|c|c|c|c|c|c|c|c|c|c|c|c|c|c|c|c|c|c|c|c|}
\hline \multirow{2}{*}{ Uber } & Android & * & * & * & 10 & * & 10 & 0 & * & 10 & 10 & 10 & * & * & * & 5 & 10 & 7,5 & 10 & 0 & 7,5 \\
\hline & iOS & - & 10 & 10 & 10 & 10 & 10 & 0 & $*$ & 10 & 10 & 10 & $*$ & * & * & 5 & - & 10 & 10 & 0 & 8,08 \\
\hline Airbnb & Android & 0 & 10 & 5 & 10 & 10 & 10 & - & * & 10 & * & 10 & * & * & * & 5 & - & 7,5 & 7,5 & 0 & 7,08 \\
\hline \multirow{2}{*}{ JustEat } & Android & - & 10 & 5 & 10 & 10 & 10 & - & * & - & 10 & 10 & * & * & * & 5 & - & 7,5 & 7,5 & 2,5 & 7,95 \\
\hline & iOS & - & 10 & 8,75 & 10 & 10 & 10 & - & $*$ & - & * & 10 & $*$ & $*$ & * & 5 & - & 10 & 10 & 0 & 8,38 \\
\hline Amazon & $\mathrm{iOS}$ & 2 & 9 & 9 & 10 & 10 & 10 & - & 10 & * & * & 10 & * & * & * & 5 & - & 10 & 10 & 0 & 7,92 \\
\hline \multirow{2}{*}{ Wallapop } & Android & * & 10 & 5 & 10 & 10 & 10 & - & * & 10 & * & 10 & * & * & * & 5 & - & 10 & 7,5 & 0 & 7,95 \\
\hline & iOS & 0 & 7,5 & 6,25 & 10 & 10 & 10 & - & 10 & * & * & 10 & * & * & * & 5 & - & 10 & 0 & 0 & 6,56 \\
\hline \multicolumn{2}{|c|}{ Media Android } & 0 & 10 & 6,5 & 10 & 10 & 10 & 3,33 & - & 10 & 10 & 10 & - & - & - & 5,45 & 10 & 8,91 & 9,1 & 0,78 & \\
\hline & Media iOS & 0,86 & 9,63 & 8,61 & 9,77 & 10 & 10 & 5 & 10 & 10 & 10 & 10 & - & - & - & 5,63 & - & 9,62 & 9,16 & 0 & \\
\hline
\end{tabular}

En la tabla podemos observar la aparición de asteriscos “*” y de guiones "_." Los asteriscos representan a aquellos indicadores que por la particularidad de las herramientas de accesibilidad que utiliza la muestra, no pueden ser evaluados. Por otro lado, los guiones, representan a aquellos indicadores que no aparecen en la App, por sus características, por tanto, no pueden ser evaluados.En consecuencia, a la hora de realizar la media de los datos de la tabla, se han omitido aquellas casillas con asterisco y guion, a fin de que no incidan negativamente sobre el resultado final.

Tras esta valoración, se ha realizado una escala de puntuación con estrellas, para que los datos puedan ser interpretados y comparados de forma visual. Desde 0 estrellas hasta 5 , desde inaccesible hasta excelente, en cuanto a nivel de accesibilidad.

Tabla 4.

Resultados del análisis experiencial del usuario

\begin{tabular}{|c|c|c|c|c|}
\hline \multirow{2}{*}{$\begin{array}{c}\text { APLICACIÓN } \\
\text { DROPBOX } \\
\end{array}$} & \multicolumn{2}{|c|}{$\begin{array}{c}\text { Grado de accesibilidad } \\
\text { Análisis experiencial del usuario } \\
\text { ANDROID }\end{array}$} & \multicolumn{2}{|c|}{$\begin{array}{c}\text { Grado de accesibilidad } \\
\text { Análisis experiencial del usuario } \\
\text { iOS }\end{array}$} \\
\hline & 玄方 & 7,5 & 玄方 & 6,98 \\
\hline SKYPE & 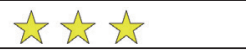 & 8,18 & $\hat{h} \hbar$ & 7,73 \\
\hline DUOLINGO & 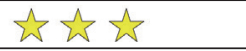 & 8,13 & 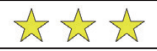 & 8,08 \\
\hline FACEBOOK & 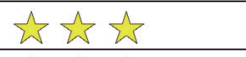 & 8,41 & 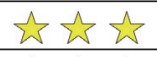 & 8,21 \\
\hline INFOJOBS & $\hat{\hbar} \hat{s} \hat{\imath}$ & 8,46 & 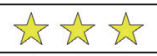 & 8,08 \\
\hline WHATSAPP & 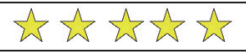 & 9,58 & 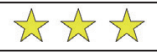 & 8,77 \\
\hline RUNTASTIC & לे ל & 7,69 & 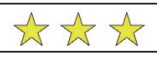 & 8,46 \\
\hline CITAPREVIASMS & 站产文 & 8,64 & 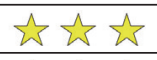 & 8,64 \\
\hline BLABLACAR & $\hat{\hbar} \hat{h}$ & 7,25 & $\hat{h} \hbar \hat{h}$ & 8,07 \\
\hline GOOGLE MAPS & 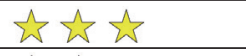 & 8,13 & 交交 & 7,73 \\
\hline MYTAXI & $\hat{h} \hat{\imath}$ & 7,5 & 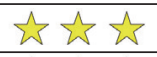 & 8,33 \\
\hline UBER & $\grave{\hbar}$ & 7,5 & $\hat{\omega} \hbar \hat{\omega}$ & 8,08 \\
\hline AIRBNB & 站论 & 7,08 & 牙交 & 7,81 \\
\hline JUSTEAT & $\hat{\Sigma} \hat{s}$ & 7,95 & 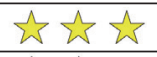 & 8,38 \\
\hline AMAZON & $\hat{\omega} \hat{\imath}$ & 7,31 & $\hbar \hat{w}$ & 7,92 \\
\hline WALLAPOP & $\grave{h} \hat{h}$ & 7,95 & $\hbar \hat{t}$ & 6,56 \\
\hline Total & 纱交 & 7,95 & 放充 & 7,99 \\
\hline
\end{tabular}

Los datos anteriores detallan el comportamiento de los sistemas operativos Android y iOS en un análisis experiencial con los 22 sujetos que han participado en el presente estudio, siendo el $51 \%$ afectados por ceguera y el $49 \%$ por baja visión.

Como se puede comprobar en los resultados finales de la tabla, en el sistema operativo iOS saca unas décimas de ventaja a la competencia, quedando iOS con un total de 8,02 y Android con un 7,95.

En general, las aplicaciones analizadas no presentan desniveles considerables entre ellas, ya que la mayoría de estas contie- nen aspectos de accesibilidad tanto positivos como negativos en igual medida.

Por un lado, encontramos que, actualmente la mayoría de las aplicaciones analizadas han presentado buenos niveles de compatibilidad con las herramientas de accesibilidad que se presentan en los dispositivos móviles, ayudando a los usuarios a poder interaccionar con estas. Al mismo tiempo, también contienen errores graves en su interfaz, presentando sesgos en la información que recibe el usuario, un ejemplo son los valores que podemos observar en la tabla 1, en cuanto al etiquetado de botones. 
Observamos una equiparación en la puntuación final, a excepción de algunos casos concretos. Pues aquellos indicadores que no pueden ser medidos, así como aquellos con una puntuación baja, compensan los valores sobresalientes del resto de indicadores

Por encima, la App que destaca de modo considerable en ambos sistemas operativos es WhatsApp, siendo algo superior en Android, con una puntuación de 9,58, que en iOS, con una puntuación de 8,77. El motivo principal es que, a diferencia de esta última, Android incorpora una medida de accesibilidad en su interfaz como es el texto ampliable.

En contraposición, la aplicación cuyo porcentaje ha sido menor es Wallapop en el sistema operativo iOS, con un valor de 6,56 . Esto se debe a las barreras de funcionalidad que ha presentado la aplicación a la hora de compatibilizar con el revisor de pantalla, que ha impedido cumplir su función elemental a los usuarios que lo han analizado.

Tabla 5.

Resultados del análisis del Entorno Personal de Accesibilidad
Nos interesa conocer cuáles son las herramientas que los usuarios de aplicaciones móviles, afectados, o bien por Ceguera, o por Baja visión, utilizan para poder acceder a la interacción con sus dispositivos móviles y con las famosas App. Para considerar una aplicación móvil accesible, los elementos de su interfaz deben ser accesibles para cualquier usuario que intente interaccionar con la App, pudiendo extraer de ella la información que anda buscando.

En el estudio que se presenta, se analizan aquellas ayudas técnicas procedentes del Sistema Operativo. Estas tienen la finalidad de transmitir el contenido que se presenta por un medio o a través de un mensaje comprensible para el usuario en cuestión. Estas herramientas recogen todos aquellos ajustes que los dispositivos móviles incorporan en los dispositivos móviles. Hablamos de la capa de accesibilidad que traen los dispositivos móviles de forma nativa en su sistema operativo.

\begin{tabular}{lccc}
\hline \multirow{2}{*}{ Herramientas } & \multicolumn{2}{c}{ Baja visión (48,98\%) } & Ceguera (51,02\%) \\
\cline { 2 - 4 } & Android (30,61\%) & iOS (18,37\%) & iOS (51,02\%) \\
\hline VoiceOver & & $18,37 \%$ & $51,02 \%$ \\
\hline VoiceAssistant & $5,1 \%$ & & \\
\hline TalkBack & $18,4 \%$ & \\
\hline Asistente Personal & $14,29 \%$ & $18,37 \%$ & \\
\hline Zoom & $24,49 \%$ & $12,24 \%$ & \\
\hline Lupa & $12,24 \%$ & & \\
\hline Ajuste de Texto & $30,61 \%$ & $18,37 \%$ & \\
\hline Ajuste de Color & $3,06 \%$ & $4,08 \%$ & $7,14 \%$ \\
\hline Ajuste de Contraste y Brillo & $3,06 \%$ & $4,08 \%$ & \\
\hline Leer Pantalla & $9,18 \%$ & & \\
\hline Braille en pantalla & & & \\
\hline
\end{tabular}

Sobre los usuarios con ceguera, en cuanto a su entorno personal de accesibilidad, podemos determinar lo siguiente:

Los usuarios con ceguera, únicamente utilizan para interaccionar con las Apps, el revisor de pantalla (VoiceOver), el asistente personal (Siri) y, la herramienta de Braille en pantalla (en un menor porcentaje). Además, hemos podido comprobar como el $100 \%$ de los usuarios con deficiencias visuales producidas por ceguera, encuestados, utilizan dispositivos con sistema operativo iOS.

Se ha comprobado que la herramienta de "asistente personal" no es una herramienta que se incluya dentro de la capa de accesibilidad de los dispositivos móviles, sin embargo, es una herramienta accesible que ayuda a la población usuaria de dispositivos móviles a sacar una mayor funcionalidad y rapidez al producto.

Esta herramienta es utilizada por la mayoría debido a la rapidez de su funcionamiento, reduciendo el manejo que se realiza con el dispositivo a un simple golpe de voz.

Por último, la herramienta "Braille en pantalla", ha sido utilizada por muy pocos usuarios. Es una herramienta difícil de manejar, la cual requiere un gran proceso de aprendizaje, sin embargo, los que si lo han utilizado, declaran su gran funcionalidad en cuanto a privacidad.

Como vemos, estas tres herramientas, citadas anteriormente, suplen los déficit de las App en accesibilidad y usabilidad, por lo que hacer aplicaciones accesibles para los usuarios con ceguera, implica hacer compatibles la interfaz de la App con las anteriores herramientas.

En cuanto a los usuarios con baja visión, podemos extraer de la anterior tabla los siguientes datos:

Los usuarios de baja visión que utilizan iOS, se deben a sus graves problemas dificultades, por ello requieren de revisor de pantalla, sobre todo, además del asistente personal, el Zoom, los ajustes de texto, de color y, de contraste y brillo.

Por otro lado, aquellos que prefieren disponer de un sistema Android, encontramos como algunos también requieren de revisor de pantalla, ya que el tamaño de la pantalla es tan reducido, que, con las ampliaciones pertinentes, la interacción no es funcional. Además, el asistente personal, el zoom, leer pantalla, la lupa, los ajustes de color y, contraste y brillo, son herramientas que utilizan con frecuencia. Todos ellos ajustan el tamaño del texto.

La utilización de revisores de pantalla, quizás es el dato más característico del entorno personal de accesibilidad del usuario, lo que nos lleva a extraer de estos datos que, debido a sus carencias visuales no se les permite interaccionar con amplificadores de manera efectiva.

La herramienta de "ajuste de texto" ha sido utilizada por todos los usuarios con baja visión, utilizando o no revisores de pantalla, por lo que, aunque se apoyen en el revisor de pantalla para funcio- 
nar mejor con el dispositivo, también requieren de datos visuales en la pantalla, y por ello, la utilización de la misma.

En definitiva, como hemos visto en los análisis anteriores, el $92,9 \%$ de la muestra analizada, requiere de un revisor de pantalla para poder acceder a las aplicaciones móviles descargadas en sus dispositivos, incluyendo los usuarios con ceguera y aquellos con baja visión, como sabemos el 49\% de la muestra representa a aquellos participantes con baja visión, y el 51\% restante a aquellos con ceguera. Este $92,9 \%$ está compuesto por un $69,4 \%$ que representa a VoiceOver, un $18,4 \%$ a TalkBack y, un 5,1\% a Voice Assistant.

Solo un $7,1 \%$ es capaz de interaccionar fructíferamente con su dispositivo móvil sin necesidad de revisores de pantalla que guíen el proceso que se lleva a cabo.

Para concluir, podemos observar que el revisor de pantalla es la herramienta más importante, con la que casi toda la población afectada dispone para poder acceder a la información que se enmarca tras el dispositivo y las Apps. Es de vital importancia atender al correcto funcionamiento de la misma, cumpliendo los indicadores de accesibilidad que dependen de esta herramienta: etiquetado de botones, acceso a los cuadros de edición y compatibilidad con la App.

\section{Referencias}

Álvarez, M. J. (2015). Smart Human City - Hacia una ciudad inteligente para todas las personas. Recuperado el 13 junio, 2018 de https://www.esmartcity.es/comunicaciones/smart-human-city-hacia-ciudad-inteligente-todas-personas

Colado, S., Gutiérrez, A., Vives, C. J y Valencia, E. (2014). Smart city. Hacia la gestión inteligente. Barcelona: Marcombo.

Directiva (UE) 2016/2102 del Parlamento Europeo y del Consejo, de 26 de octubre de 2016, sobre la accesibilidad de los sitios web y aplicaciones para dispositivos móviles de los organismos del sector público. Diario Oficial de la Unión Europea L 327, 2 de diciembre de 2016, pp 1-15. Cita: Dir. 2016/2102 del Consejo, de 26 de octubre de 2018

Discapnet. (2009). Discapnet: El portal de las personas con discapacidad. Recuperado el 11 febrero, 2015 de http://www.discapnet. es/Castellano/Paginas/default.aspx

Gil, S. y Rodríguez-Porrero, C. (2013). Cómo hacer "Apps" accesibles". Infórmate. Madrid: CEAPAT-IMSERSO. Recuperado el 21 Enero, 2015, de http://www.ceapat.es/InterPresent1/ groups/imserso/documents/binario/Appsaccesibles.pdf

Hassan, Y. y Martín, F. J. (2003). Qué es la Accesibilidad Web. No solo usabilidad: revista sobre personas, diseño y tecnología. Recuperado el 15 enero, 2015, de http://www.nosolousabilidad.com/ articulos/accesibilidad.htm?iframe $=$ true\&width $=90 \% \&$ height $=90 \%$

Olmedo-Moreno, E. V. y López-Delgado, A. (2015). De la Smart City a la Smart Human City. Inclusión digital en aplicaciones. Revista Fuentes, 17 (Diciembre), 41-65. [Fecha de consulta: 14/12/2017]. doi: http://dx.doi.org/10.12795/revistafuentes.2015.i17.02.

Web Content Accessibility Guidelines (WCAG) 2.1. (2017) Ben Caldwell; Michael Cooper; Loretta Guarino Reid; Gregg Vanderheiden et al. W3C. 11 December 2008. W3C Recommendation. URL: https://www.w3.org/TR/WCAG20/ 
